\title{
Social sorting
}

Citation for published version (APA):

Cowan, R., \& Jonard, N. (2003). Social sorting. UNU-MERIT, Maastricht Economic and Social Research and Training Centre on Innovation and Technology. MERIT-Infonomics Research Memorandum Series No. 032 https://doi.org/10.26481/umamer.2003032

\section{Document status and date:}

Published: 01/01/2003

DOI:

10.26481/umamer.2003032

Document Version:

Publisher's PDF, also known as Version of record

\section{Please check the document version of this publication:}

- A submitted manuscript is the version of the article upon submission and before peer-review. There can be important differences between the submitted version and the official published version of record.

People interested in the research are advised to contact the author for the final version of the publication, or visit the DOI to the publisher's website.

- The final author version and the galley proof are versions of the publication after peer review.

- The final published version features the final layout of the paper including the volume, issue and page numbers.

Link to publication

\footnotetext{
General rights rights.

- You may freely distribute the URL identifying the publication in the public portal. please follow below link for the End User Agreement:

www.umlib.nl/taverne-license

Take down policy

If you believe that this document breaches copyright please contact us at:

repository@maastrichtuniversity.nl

providing details and we will investigate your claim.
}

Copyright and moral rights for the publications made accessible in the public portal are retained by the authors and/or other copyright owners and it is a condition of accessing publications that users recognise and abide by the legal requirements associated with these

- Users may download and print one copy of any publication from the public portal for the purpose of private study or research.

- You may not further distribute the material or use it for any profit-making activity or commercial gain

If the publication is distributed under the terms of Article $25 \mathrm{fa}$ of the Dutch Copyright Act, indicated by the "Taverne" license above, 


\section{MERIT-Infonomics Research Memorandum series}

\section{Social Sorting}

Robin Cowan \& Nicolas Jonard

2003-032
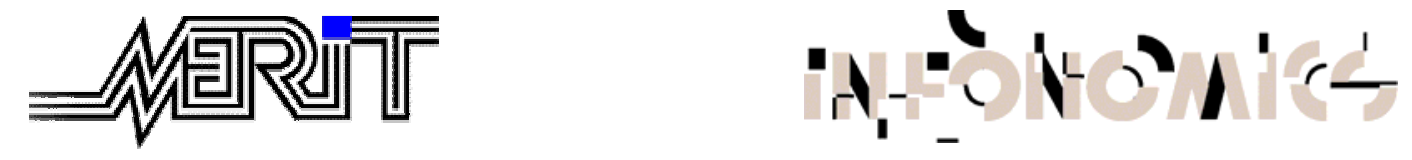

MERIT - Maastricht Economic Research

International Institute of Infonomics

Institute on Innovation and Technology

PO Box 616

6200 MD Maastricht

The Netherlands

T: +31433883875

F: +31 433884905

http://www.merit.unimaas.nl

e-mail:secr-merit@merit.unimaas.nl

c/o Maastricht University

PO Box 616

6200 MD Maastricht

The Netherlands

T: +31433883875

F: +31453884905

http://www.infonomics.nl e-mail: secr@infonomics.nl 


\title{
Social Sorting*
}

\author{
R. COWAN ${ }^{\dagger}$ N. JONARD ${ }^{\dagger}$ \\ ${ }^{\dagger}$ MERIT, University of Maastricht, P.O. Box 616 \\ 6200 MD Maastricht, The Netherlands \\ ${ }^{\ddagger}$ CNRS, CREA, Ecole Polytechnique, 1 Rue Descartes \\ 75005 Paris, France
}

December 1, 2003

\begin{abstract}
This paper is about the behaviour of a society in which learning individuals play a Prisoner's Dilemma that guides social promotion and demotion. The direct effect of the payoff-based socialization that is implemented here is segregation. However, segregation permits the survival of cooperation as it (unintendedly) preserves cooperators from detrimental interactions with defectors. Very large amounts of cooperation can be observed in the long run.
\end{abstract}

JEL Classification: C70, C72, C73, D72

Keywords: Cooperation, Socialization, Imitation

\section{Introduction}

In many circumstances the people with whom we interact are not drawn randomly from the population as a whole. Indeed, there tends to be some relatively small group to which each person belongs and with whom he or she interacts most frequently. Often this grouping feature can be described as constituting a social hierarchy, in which we interact mostly with people near our level in the hierarchy. The hierarchy changes though. Agents can move up or down, and as they do, those with whom they interact change. One might say that this effect is the whole point of moving within the hierarchy. Promotion or demotion,

*Corresponding author: r.cowan@merit.unimaas.nl; Tel.: 3143388 3878; Fax: 31433884905 
from one group to the next is very often determined by one's standing within one's current group. If an agent falls to the bottom of his group, he may be shunned by other members as "not quite the thing". Demotion may be appealing (becoming a bigger fish in a smaller pond), ${ }^{1}$ or it may be necessary as current group members refuse to interact any longer. On the other hand rising to the top of a group may create the possibility of moving up either due to the Groucho Marx effect (do I want to be part of a club that would have me as a member?); or through invitation from above, to replace someone who has been demoted.

How does an agent choose behaviour in such a world? One thing about a social hierarchy, as opposed to social clubs, is that while social clubs try hard to create opaque walls, social hierarchies tend to have transparent walls. In other words, while it can be difficult to observe actions with clubs other than one's own, actions of agents in different places in the social hierarchy tend to be quite visible. This implies that an agent has a relatively wide vision when deciding which of the available actions will be effective. Agents will look at the entire population, rather than just locally, to decide which strategies to employ. In addition, the possibility of promotion or demotion suggests that looking beyond one's social group can be a more effective long-term learning strategy, since one will carry one's behaviour along, when changing groups if that happens.

The model developed in this paper addresses the joint effects and socialization and learning on the welfare of a finite-sized population of individuals repeatedly playing a Prisoner's Dilemma (PD). There is a large recent literature on local interaction in population games wherein the focus has been on interaction structures that can support cooperation as a stochastically stable equilibrium. ${ }^{2}$ This work tends to impose an interaction structure and then invite agents to learn about which strategies to employ. Several learning mechanisms have been proposed, a common one being imitative learning. Vega-Redondo (2000), in the context of a Coordination Game, introduces the notion of an interaction structure that evolves through changing social hierarchies. Players play only those within the same social group, but the social structure can evolve as agents move up or down the hierarchy. ${ }^{3}$

This paper combines these ideas: imitative learning, local interaction and social evolution, all in the context of the repeated one-shot PD. Interaction is local in that a player plays only with the other members of his social group, but learning is global in that it is

\footnotetext{
${ }^{1}$ See Frank (1987) on "choosing the right pond".

${ }^{2}$ On the general importance of local interaction structures see Anderlini and Ianni (1996); Durlauf (1993); Berninghaus and Schwalbe (1996); Blume (1993); Ellison (1993); regarding the Prisoner's Dilemma see Eshel et al. (1998); Nowak and May (1992); Nowak et al. (1994) and references therein.

${ }^{3}$ Vega-Redondo acknowledges that he was inspired by Harrington (1998) in the idea of an evolving social hierarchy.
} 
based on the payoffs of play in the entire population.

Social evolution - or "socialization" for convenience - takes the form of a payoff-based promotion mechanism which reshuffles individuals across social groups (we will indifferently use the term social locations) but keeps the population composition of strategies unchanged. Learning on the other hand dictates strategy change and therefore governs the evolution of the population composition. The two mechanisms operate in parallel. Specifically, we assume that each time period one individual is picked at random and given the chance to be promoted by swapping group with someone located higher in the social structure, and then one individual (possibly the same) is given a learning draw on the basis of which he/she possibly revises strategy. These changes are subject to small independent random perturbations (mutations) and our interest is in the behaviour of the system when mutations become arbitrarily small.

This structure permits us to examine situations in which interaction is sufficiently local that a cohesive group can form, and thus preserve cooperation. Interestingly, however, cooperation is preserved not only as the unique strategy played within this group, but can in general spill over into other groups that also contain defectors. What makes this possible is that social "sorting" effectively expels defectors from the group of cooperators, as they are "kicked upstairs". ${ }^{4}$ The sorting that removes the highly-paid defectors from a group of cooperators and forces them to play with other defectors, eventually equalizes the payoffs of the two strategies, and cooperation is sustained.

\section{The model}

This section is devoted to the presentation of the main hypothesis and the first preliminary results of the model.

\subsection{Assumptions}

Let $S=\{1, \ldots, n\}$ denote a finite population of individuals. Time is discrete and indexed with $t=0,1, \ldots$ Players repeatedly engage in the one-shot Prisoner's Dilemma with payoffs given in Table 1.

Payoffs are assumed to satisfy $0<b<1 / 2$, which implies that defection is a dominant strategy in the one-shot game, and that cooperation is also the only symmetric and efficient

\footnotetext{
${ }^{4}$ This is related to the mechanism that supports knowledge sharing within a coalition in Eaton and Eswaran (1997), in which defectors are expelled. In the current model defectors are sent to another group whereas in Eaton and Eswaran they are forever black-listed from all groups.
} 


\begin{tabular}{r|c|c|} 
Row $\backslash$ Col & \multicolumn{1}{c}{$C$} & \multicolumn{1}{c}{$D$} \\
\cline { 2 - 3 }$C$ & 1 & 0 \\
\cline { 3 - 3 }$D$ & $1+b$ & $b$ \\
\cline { 2 - 3 } & &
\end{tabular}

Figure 1: Payoffs to the row player for the two-by-two symmetric Prisoner's Dilemma

outcome (it dominates any symmetric mixing of cooperation and defection).

There are 2 locations of identical size $s \geq 2$ with $2 s=n$. The locations can be interpreted as ordered according to some (commonly agreed upon) social dimension. They are occupied by the same number of individuals and it is assumed that location 2 corresponds to a higher position in the social hierarchy than location 1 . Let $\ell(i)$ denote the location of individual $i \in S$. Define $n_{D, \ell}$ to be the number of $D$-players in location $\ell=1,2$ (hence $s-n_{D, \ell}$ is the number of $C$ players at that location), and let $n_{D}$ denote the total number of defectors in the population, $n_{D}=n_{D, 1}+n_{D, 2}$. The state of the system at any time $t=0,1, \ldots$ is fully described by the 2 -dimensional vector $\left(n_{D, 1}, n_{D, 2}\right)$ providing the number of defectors in each location.

The dynamics of the system are as follows.

1. Play. At each time period, each individual engages in a tournament with all the individuals at his location including himself, applying uniformly the same action $C$ or $D$ to all his opponents. The outcome of this round of play is used to guide social promotion.

2. Socialization. At each time period after play has taken place, an individual who realizes the highest payoff in location 1 is picked up at random. With probability $1-\epsilon$ he swaps location with an individual who realizes the lowest payoff in location 2 , whereas with probability $0 \leq \epsilon<1$ nothing changes. Promotion is based on wealth, and it is simply assumed that the wealthiest individual of social location 1 is promoted, while the poorest individual from location 2 is demoted.

3. Imitation. After play and socialization have been completed, an individual is picked up at random in any location. He compares the global performance statistics associated with actions $C$ and $D$ and adopts the best action with probability $1-\epsilon$, whereas the other action is chosen with probability $0 \leq \epsilon<1$. Action performance is measured by the average payoff produced over all the meetings in which the action is involved.

The system evolves through socialization and learning, and both mechanisms are continuously subject to small random perturbations captured by $\epsilon$. Our concern is with the 
system's most likely outcomes, i.e. states in which it almost surely rests when $\epsilon$ goes to zero.

\section{$2.2 \quad$ Properties}

The random sequence $\left\{w^{\epsilon, t} \equiv\left(n_{D, 1}^{\epsilon, t}, n_{D, 2}^{\epsilon, t}\right) ; t \geq 0\right\}$ is a stationary Markov process, with finite state space $\Omega \equiv\{0, \ldots, s\}^{2}$. Let the probability of a one-step transition from state $w=\left(n_{D, 1}, n_{D, 2}\right)$ to state $w^{\prime}=\left(n_{D, 1}^{\prime}, n_{D, 2}^{\prime}\right)$ be written as $p^{\epsilon}\left(w, w^{\prime}\right)$ for all $w, w^{\prime} \in \Omega$. Let $P^{\epsilon}$ denote the corresponding transition matrix.

Proposition 1 The stochastic process $\left\{w^{\epsilon, t} ; t \geq 0\right\}$ possesses a unique stationary distribution $\mu^{\epsilon}$ for all $\epsilon>0$.

Proof. The process $\left\{w^{\epsilon, t} ; t \geq 0\right\}$ possesses a uniquely defined stationary distribution provided it is irreducible and ergodic (cf. Ross, 1992). Irreducibility stems from having all states in $\Omega$ communicating. Indeed assuming socialization does not take place (which occurs with positive probability when $\epsilon>0$ ) there exists a sequence of best responses (with probability $1-\epsilon$ ) and errors (with probability $\epsilon$ ) such that any state can be reached from any other state. Ergodicity obtains if $\left\{w^{\epsilon, t} ; t \geq 0\right\}$ is positive recurrent and aperiodic, both conditions being always satisfied for a finite irreducible Markov chain.

The stationary distribution $\mu^{\epsilon}$ satisfies $\mu^{\epsilon} P=\mu^{\epsilon}$ and $\sum_{w} \mu^{\epsilon}(w)=1$. Rather than studying it directly, we shall use the methodology developed by Freidlin and Wentzell (1984), which involves determining absorbing sets and their basins of attraction for the mutation free process $(\epsilon=0)$ and then examining stochastic stability by counting mutations. (See Young, 1998 for extremely clear presentation and applications of the technique.)

\section{Absorbing sets of the mutation free dynamics}

An absorbing, or stationary, state is a state from which, once it is reached, the system never exits. Absorbing in this model is with respect to both socialization and learning. Therefore we start by identifying stationary states under (mutation free) promotion and then we single out the subset of elements of this set that are also stationary under the (mutation free) learning dynamics. Two obvious absorbing states of the process are the convention in which everybody defects, $w_{D}=(s, s)$, and the convention in which everybody cooperates, $w_{C}=(0,0)$. Once $w_{D}$ or $w_{C}$ is reached, neither socialization (there is no swapping taking place, or more precisely, swapping has no effect on $\left.w \equiv\left(n_{D, 1}, n_{D, 2}\right)\right)$ nor imitation (only one strategy is present) can get the system to leave it. 


\subsection{Socialization}

The population composition is fixed ( $n_{D}$ is constant, there is no imitation) and individuals are only being re-allocated across social groups by the mutation free promotion/demotion mechanism. The following result is easily shown.

Proposition 2 Only a configuration having at most one mixed location can be absorbing under the postulated socialization mechanism. The mixed location is either below a pure D location - in the form $\left(n_{D, 1}, s\right)$ - or above a pure $C$ location - in the form $\left(0, n_{D, 2}\right)$.

Proof. Consider an absorbing state $w$. First note that $w$ cannot be in the form $\left(n_{D, 1}, 0\right)$, i.e. such that there is a pure $C$ location above a mixed one, as a $D$ from the mixed location would be promoted and the pure $C$ location would be destroyed. For the same reason $w$ cannot be in the form $\left(s, n_{D, 2}\right)$, i.e. a pure $D$ location below a mixed group, since a $C$ would be demoted and the pure $D$ group would be destroyed. Similarly, two mixed groups is not feasible since $D$ s would be promoted and $C$ s demoted until one or both of the mixed groups is destroyed and becomes a group of agents all playing the same strategy (in a mixed group defection dominates).

Consider now the issue of learning.

\subsection{Imitation}

Let $\Pi_{D}$ be the average payoff to $D$ players, let $\Pi_{C}$ be the average payoff to $C$ players. In state $w=\left(n_{D, 1}, n_{D, 2}\right)$, one has

$$
\Pi_{C}=\Pi_{C}(w)=\frac{\sum_{\ell=1}^{2}\left(s-n_{D, \ell}\right)^{2}}{n-n_{D}}
$$

and

$$
\Pi_{D}=\Pi_{D}(w)=\frac{\sum_{\ell=1}^{2} n_{D, \ell}\left[b n_{D, \ell}+(1+b)\left(s-n_{D, \ell}\right)\right]}{n_{D}} .
$$

Given the previous section, we know that if $w$ is an absorbing state with regard to socialization it is in the form $\left(0, n_{D, 2}\right)$ or $\left(n_{D, 1}, s\right)$. It is therefore enough to consider two regions for $n_{D}$ the total number of defectors: $1 \leq n_{D} \leq s$ and $s+1 \leq n_{D} \leq n$. It will also be convenient, though $n_{D}$ is an integer number, to treat it formally as a scalar.

In the first region $1 \leq n_{D} \leq s$, the world consists of a mixed group at location 2 and a pure cooperation group at location 1, i.e. $w=\left(0, n_{D}\right)$. Equations 1 and 2 reduce to 
$\pi_{D}\left(n_{D}\right)=b n_{D}+(1+b)\left(s-n_{D}\right)$ and $\pi_{C}\left(n_{D}\right)=\left[s^{2}+\left(s-n_{D}\right)^{2}\right] /\left(n-n_{D}\right)$. Equating the two expressions and noting that $n=2 s$ yields a unique solution

$$
n_{D}^{-}=\frac{2 b s}{1+b} .
$$

There is, thus, a unique sign change for the payoff difference $\pi_{D}\left(n_{D}\right)-\pi_{C}\left(n_{D}\right)$ in $1 \leq$ $n_{D} \leq s$. As the payoff difference is a continuous function over the interval considered, it remains to compute $\pi_{D}(1)-\pi_{C}(1)=s(2 b s-b-1) /(2 s-1)$, This is larger than 0 when $b>1 /(2 s-1)$, a property we would expect to hold in general when $s$ is large. Summarizing, the equation $\Pi_{D}\left(n_{D}\right)=\Pi_{C}\left(n_{D}\right)$, has a unique root $1 \leq n_{D}^{-} \leq s$, and the payoff to $D$ is smaller than $\Pi_{C}$ when $n_{D}>n_{D}^{-}$, while it is larger when $n_{D}<n_{D}^{-}$. In the region $1 \leq n_{D} \leq s$ the pure (mutation free) learning dynamics would therefore drive the process to $w_{D}^{-}=\left(0, n_{D}^{-}\right)$if $n_{D}^{-}$is an integer number, and to $\left\{\left(0,\left\lfloor n_{D}^{-}\right\rfloor\right),\left(0,\left\lceil n_{D}^{-}\right\rceil\right)\right\}$otherwise. In the rest of the paper it is assumed that $n_{D}^{-}$is not an integer number, hence the absorbing set is defined $S_{D}^{-} \equiv\left\{\left(0,\left\lfloor n_{D}^{-}\right\rfloor\right),\left(0,\left\lceil n_{D}^{-}\right\rceil\right)\right\}$.

Regarding the region $s+1 \leq n_{D} \leq n$, the only candidate for a stable state is in the form $w=\left(n_{D} \bmod s, s\right)$, i.e. a mixed group in location 1 and a pure defection group above. Use $n_{C}=n-n_{D}$ as the argument: then $\pi_{D}\left(n_{C}\right)=\left(2 s^{2} b-b s n_{C}+n_{C} s-n_{C}^{2}\right) /\left(n-n_{C}\right)$ and $\pi_{C}\left(n_{C}\right)=n_{C}$. Setting $\pi_{D}\left(n_{C}\right)=\pi_{C}\left(n_{C}\right)$ yields a unique

$$
n_{C}^{*}=\frac{2 b s}{1+b},
$$

and hence a unique sign change for the difference $\pi_{D}\left(n_{C}\right)-\pi_{C}\left(n_{C}\right)$ for $1 \leq n_{C} \leq s$ (this root for $n_{C}$ is the same as the one for $n_{D}$ in the previous case). It remains to compute $\pi_{D}(1)-\pi_{C}(1)=s(1-b+2(b s-1)) /(2 s-1)$, which is again larger than 0 when $b>1 /(2 s-1)$. Now define $n_{D}^{+}=n-n_{C}^{*}$. Summarizing we have a unique root $n_{D}^{+} \in\{s+1, n\}$ satisfying $\Pi_{D}\left(n_{D}\right)=\Pi_{C}\left(n_{D}\right)$, and the payoff to $C$ is larger than to $D$ when $n_{D}<n_{D}^{+}$, while it is lower when $n_{D}>n_{D}^{+}$. In the region $\{s+1, n\}$ the pure (mutation free) learning dynamics would therefore drive the process away from $w_{D}^{+}=\left(n_{D}^{+} \bmod s, s\right)$. To simplify matters, it is assumed hereafter that $n_{D}^{+}$is not an integer.

From the previous discussion the following proposition is derived.

Proposition 3 The absorbing sets of the process $\left\{w^{0, t} ; t \geq 0\right\}$ are the convention in which everybody defects $w_{D}=(s, s)$, the convention in which everybody cooperates $w_{C}=(0,0)$, and the low defection mixed set $S_{D}^{-}$as it has been identified in the previous discussion.

Proof. The essential elements have been presented above. The last step is to determine the behaviour of the mutation free process starting from any possible state $\left(n_{D, 1}, n_{D, 2}\right) \in \Omega$. 


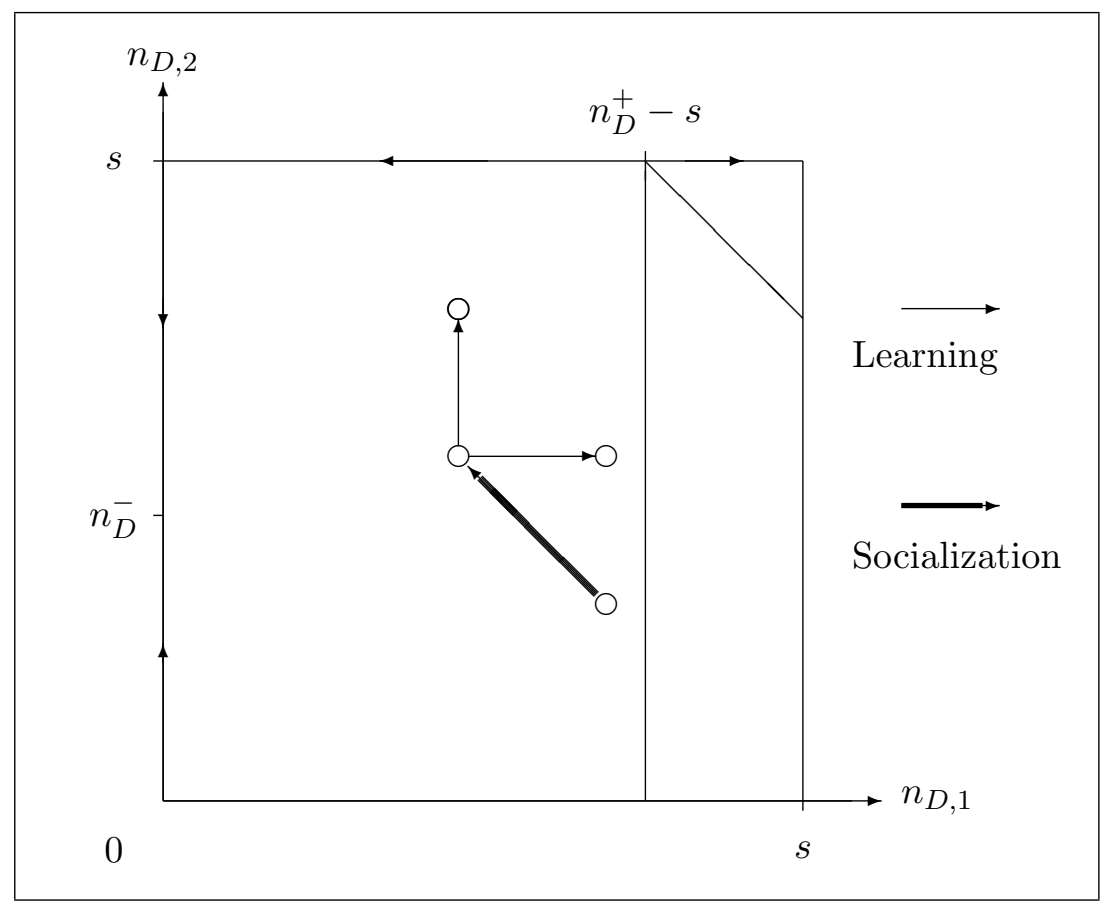

Figure 2: The state space $\Omega$ and the directions of motion of socialization and mutation free learning.

In Figure 2 we depict the state space and the possible directions of motion of the mutationfree process $\left\{w^{0, t} ; t \geq 0\right\}$. Consider a interior state $\left(n_{D, 1}, n_{D, 2}\right)$ with $n_{D, 1}>0$ and $n_{D, 2}<s$. Socialization promotes a defector and demotes a cooperator, so in Figure 2 this process moves the system north-west. Defection dominates in both groups so learning with certainty moves the system either north or east. Thus $n_{D, 2}$ is monotonically increasing while $n_{D, 1}$ either stays constant of decreases. These two remarks imply that after a finite number of steps the system necessarily reaches either a state in the form $\left(n_{D, 1}^{\prime}, s\right)$ with $n_{D, 1}^{\prime} \leq n_{D, 1}$, or a state in the form $\left(0, n_{D, 2}^{\prime}\right)$ with $n_{D, 2}^{\prime}>n_{D, 2}$. In these states there is at most one mixed location, and locations are properly sorted. What happens once it is there?

First assume we reach a state $\left(n_{D, 1}^{\prime}, s\right)$, with $n_{D, 1}^{\prime} \leq n_{D, 1}$. Either $n_{D, 1}^{\prime}>n_{D}^{+}-s$ and then learning implies that the number of defectors in location 1 increases until $w_{D}=(s, s)$ is eventually reached. Or $n_{D, 1}^{\prime}<n_{D}^{+}-s$ and then learning implies that the number of defectors in location 1 decreases to 0 . Second assume we reach a state $\left(0, n_{D, 2}^{\prime}\right)$ with $n_{D, 2}^{\prime}>n_{D, 2}$. Whatever the value of $n_{D, 2}^{\prime}$ learning drives the process to $S_{D}^{-}$.

The conclusion is that from any state $\left(n_{D, 1}, n_{D, 2}\right)$ such that $0<n_{D, 1}<n_{D}^{+}-s$ the system eventually reaches $S_{D}^{-}$. These states constitute the basin of attraction of $S_{D}^{-}$, and can be denoted $\mathbf{B}\left(S_{D}^{-}\right)$. (It is obvious that $\mathbf{B}\left(w_{C}\right)=\left\{w_{C}\right\}$.) Can we characterize $\mathbf{B}\left(w_{D}\right)$ ? 
It is simply defined by the constraint $n_{D, 1}+n_{D, 2}>n_{D}^{+}$, which is the triangle in the upper right corner of $\Omega$ in Figure 2. To see this, consider that the only way to move west is through socialization. But this also moves the system north. Inside the triangle $\mathbf{B}\left(w_{D}\right)$, it is not possible to move far enough west to arrive at $n_{D, 1}<n_{D}^{+}-s$ before arriving at $n_{D, 2}=s$. Thus starting within this triangle leads the system to $w_{D}$. The remaining trapezoid in the figure has the property that either $\mathbf{B}\left(S_{D}^{-}\right)$or $\mathbf{B}\left(w_{D}\right)$ can be reached from it.

In the next section we move to stochastic stability.

\section{Stochastic stability}

At this stage two results are useful (cf. Young, 1993; Kandori et al., 1993). The first is that if $P^{\epsilon}$ is a regular perturbed Markov process, then $\lim _{\epsilon \rightarrow 0} \mu^{\epsilon}=\mu^{0}$ exists and is a stationary distribution of $P^{0}$. The second result is that the stochastically stable states of the process are contained in the recurrent class(es) of $P^{0}$ having minimum stochastic potential.

That the process considered here is indeed a regular perturbed Markov process is easily seen. First, we have seen that $P^{\epsilon}$ is irreducible for every $\epsilon>0$ (any two states communicate). Second, we trivially have that $\lim _{\epsilon \rightarrow 0} p^{\epsilon}\left(w, w^{\prime}\right)=p^{0}\left(w, w^{\prime}\right)$. Third and last, for any pair $\left(w, w^{\prime}\right)$ such that the one step transition probability $p^{\epsilon}\left(w, w^{\prime}\right)$ is strictly larger than 0 for $\epsilon>0$, there exists an integer number $r\left(w, w^{\prime}\right) \geq 0$ such that $0<$ $\lim _{\epsilon \rightarrow 0} p^{\epsilon}\left(w, w^{\prime}\right) / \epsilon^{r\left(w, w^{\prime}\right)}<\infty$. Indeed the one step transition probabilities are all products involving $\epsilon$ or $(1-\epsilon)$. The number $r\left(w, w^{\prime}\right)$ is called the resistance of the transition from $w$ to $w^{\prime}$. Transitions that can occur in the mutation-free process have zero resistance and $r\left(w, w^{\prime}\right)=\infty$ if $p^{\epsilon}\left(w, w^{\prime}\right)=p^{0}\left(w, w^{\prime}\right)=0$ for all $\epsilon>0$.

Regarding stochastic stability, the first step has been to determine the recurrent (or absorbing) sets for the mutation free process $P^{0}$. They are three and were identified in the previous section. Step two now consists in determining the stochastic potential of the three absorbing sets. For this purpose the space of directed graphs having the recurrent sets as vertices is considered. The stochastic potential of a recurrent set is the minimum resistance over all trees rooted at this particular set. The resistance of a rooted tree is the sum of the edge resistances on the two edges that compose it. Finally the resistance of a transition from one absorbing set to another is the least resistance over all paths (sequence of states) that start in the former and end in the latter. There are 2 absorbing states $w_{C}$ and $w_{D}$, and the absorbing set $S_{D}^{-}$. For each of them we need to construct the 3 possible rooted trees and identify the one with minimum resistance. With the help of Figure 2 this is easily done, and yields the following proposition. 
Proposition 4 The stochastically stable set of the process $\left\{w^{0, t} ; t \geq 0\right\}$ is the low defection set $S_{D}^{-}$as long as $\left\lfloor n_{D}^{-}\right\rfloor<s / 2$. The process admits two stochastically stable sets when $\left\lfloor n_{D}^{-}\right\rfloor=$ $s / 2$, and finally the stochastically stable state is overall defection $w_{D}$ when $\left\lfloor n_{D}^{-}\right\rfloor>s / 2$. Equivalently, as long as $b<1 / 3$ the long run outcome of the joint process of socialization and learning is a population consisting of $\lfloor n /(1+b)\rfloor$ altruists. The stochastically stable state with the lowest amount of cooperation (apart from $w_{D}$ ) has $75 \%$ of cooperators and obtains when $b=1 / 3$. If the bonus to defection $b$ is larger than $1 / 3$, the stochastically stable state has only defectors.

Proof. Start with trees rooted at $w_{C}$. The 3 trees are

$$
\begin{aligned}
& w_{D} \rightarrow w_{C} \leftarrow S_{D}^{-}, \\
& w_{D} \rightarrow S_{D}^{-} \rightarrow w_{C}, \\
& S_{D}^{-} \rightarrow w_{D} \rightarrow w_{C} .
\end{aligned}
$$

The stochastic potential of $w_{C}$ is denoted $\gamma_{C}$. It is the minimum of $\rho\left(w_{D}, w_{C}\right)+\rho\left(S_{D}^{-}, w_{C}\right)$, $\rho\left(w_{D}, S_{D}^{-}\right)+\rho\left(S_{D}^{-}, w_{C}\right)$ and $\rho\left(S_{D}^{-}, w_{D}\right)+\rho\left(w_{D}, w_{C}\right)$. From Figure 2 it is straightforward to see that the tree with minimum resistance is $w_{D} \rightarrow S_{D}^{-} \rightarrow w_{C}$, for which $\rho\left(w_{D}, S_{D}^{-}\right)=n-$ $\left\lfloor n_{D}^{+}\right\rfloor$and $\rho\left(S_{D}^{-}, w_{C}\right)=\left\lfloor n_{D}^{-}\right\rfloor$. Hence $\gamma_{C}=n-\left\lfloor n_{D}^{+}\right\rfloor+\left\lfloor n_{D}^{-}\right\rfloor$. Let $\gamma_{D}$ denote the stochastic potential of $w_{D}$ and let $\gamma_{D}^{-}$that of $S_{D}^{-}$. We do the same computation as before on

$$
\begin{aligned}
& w_{C} \rightarrow w_{D} \leftarrow S_{D}^{-}, \\
& w_{C} \rightarrow S_{D}^{-} \rightarrow w_{D}, \\
& S_{D}^{-} \rightarrow w_{C} \rightarrow w_{D} .
\end{aligned}
$$

$\gamma_{D}$ is the minimum of $\rho\left(w_{C}, w_{D}\right)+\rho\left(S_{D}^{-}, w_{D}\right), \rho\left(w_{C}, S_{D}^{-}\right)+\rho\left(S_{D}^{-}, w_{D}\right)$ and $\rho\left(S_{D}^{-}, w_{C}\right)+$ $\rho\left(w_{C}, w_{D}\right)$. Obviously the least resistant $w_{D^{-}}$-tree is $w_{C} \rightarrow S_{D}^{-} \rightarrow w_{D}$, with resistance $\rho\left(w_{C}, S_{D}^{-}\right)+\rho\left(S_{D}^{-}, w_{D}\right)=\gamma_{D}=1+\left\lceil n_{D}^{+}\right\rceil-s+1$. Finally consider $S_{D}^{-}$-trees. They are

$$
\begin{aligned}
& w_{C} \rightarrow S_{D}^{-} \leftarrow w_{D}, \\
& w_{C} \rightarrow w_{D} \rightarrow S_{D}^{-}, \\
& w_{D} \rightarrow w_{C} \rightarrow S_{D}^{-} .
\end{aligned}
$$

The least resistant $S_{D}^{-}$-tree is $w_{C} \rightarrow S_{D}^{-} \leftarrow w_{D}$, with resistance $\gamma_{D}^{-}=1+n-\left\lfloor n_{D}^{+}\right\rfloor$. It is now possible to examine stochastic stability by finding the absorbing set having minimum stochastic potential. These are

$$
\begin{aligned}
\gamma_{C} & =n-\left\lfloor n_{D}^{+}\right\rfloor+\left\lfloor n_{D}^{-}\right\rfloor, \\
\gamma_{D} & =2+\left\lceil n_{D}^{+}\right\rceil-s, \\
\gamma_{D}^{-} & =1+n-\left\lfloor n_{D}^{+}\right\rfloor .
\end{aligned}
$$


Now note that $n_{D}^{+}+n_{D}^{-}=n$ entails $\left\lfloor n_{D}^{+}\right\rfloor+\left\lceil n_{D}^{-}\right\rceil=n$, hence

$$
\begin{aligned}
& \gamma_{C}=2\left\lfloor n_{D}^{-}\right\rfloor+1, \\
& \gamma_{D}=2+s-\left\lfloor n_{D}^{-}\right\rfloor, \\
& \gamma_{D}^{-}=2+\left\lfloor n_{D}^{-}\right\rfloor .
\end{aligned}
$$

At this stage it is clear that as soon as $\left\lfloor n_{D}^{-}\right\rfloor>1$ the stochastic potential of $w_{C}$ exceeds $\gamma_{D}^{-}$. So the competition is between $S_{D}^{-}$and $w_{D}$, i.e. the objective is to compare $s-\left\lfloor n_{D}^{-}\right\rfloor$and $\left\lfloor n_{D}^{-}\right\rfloor$, or $\left\lfloor n_{D}^{-}\right\rfloor$and $s / 2$. Working directly on the algebraic expression $2 s b /(1+b)=s / 2$ yields $b=1 / 3$ hence the proposition.

\section{Conclusion}

In this paper we have examined the joint effects of socialization and learning on cooperation, and have shown that a payoff-based socialization rule is likely to sustain large amounts of cooperation. The intuition is the following. In general cooperation survives when played within closed (or cohesive enough) sub-groups (see for instance local iteration models à la Eshel, Samuelson and Shaked, 1998 or Nowak and May, 1992), and when the same is true for defection. In this way the payoff from cooperation tends to be close to 1 , while that to defection tends to be close to $b<1$. The modelling issue therefore is to find of way of getting $C$ s and $D$ s to segregate while preserving the global visibility of payoffs. Local interactions produce that feature by imposing a fixed neighbourhood structure. In this paper we take a different path by considering an assortative social promotion mechanism. It is a natural and reasonable one as it assumes promotion for efficient players and demotion for those implementing poorly rewarding strategies. It does produce segregation while it preserves a wide observability of payoffs. As a result cooperation has a chance to generate large payoffs and be observed, and therefore further expand. This is true for reasonable values of the premium to defect. Assortative matching therefore appears as a way to sustain cooperative behaviour.

\section{References}

Anderlini, L. and Ianni, A. (1996) Path Dependence and Learning from Neighbors. Games and Economic Behavior, 13, 141-158.

Durlauf, S. (1993) Non-ergodic Economic Growth, Review of Economic Studies, 60, 349366. 
Berninghaus, S. and Schwalbe, U. (1996) Evolution, Interaction and Nash Equilibria. Journal of Economic Behavior and Organization, 29, 57-85.

Blume, L. (1993) The Statistical Mechanics of Strategic Interaction. Games and Economic Behavior, 5, 387-424.

Eaton, C. and M. Eswaran, (1998) Endogenous Cartel Formation, Australian Economic Papers, 37, 1-13.

Ellison, G. (1993) Learning, local interaction and coordination. Econometrica, 61, 10471071.

Eshel, I. and Samuelson, L. and Shaked, A. (1998) Altruists, Egoists and Hooligans in a Local Interaction Model. American Economic Review, 88, 157-179.

Frank, R. (1985) Choosing the Right Pond : Human Behavior and the Quest for Status Oxford University Press, Oxford.

Freidlin, M. and Wentzell, A. (1984) Random Perturbations of Dynamical Systems. SpringerVerlag, Berlin.

Harrington, J.E. (1998) The Social Selection of Flexible and Rigid Agents. American Economic Review, 88, 63-82.

Kandori, M., Mailath, G. and Rob, R. (1993) Learning, Mutation and Long Run Equilibria in Games. Econometrica, 61, 29-56.

Nowak, M. and May, R. (1992) Evolutionary Games and Spatial Chaos. Nature, 359, 826-829.

Nowak, M., Bonhoeffer, S. and May, R. (1994) Spatial Games and the Maintenance of Cooperation. Proceedings of the National Academy of Science USA, 91, 4877-4881.

Robson, A. J. and Vega-Redondo, F. (1996) Efficient Equilibrium Selection in Evolutionary Games with Random Matching. Journal of Economic Theory, 70, 65-92.

Ross, S. (1992) Introduction to Probability Models. Academic Press, 5th. edition.

Vega-Redondo, F. (2000) Unfolding Social Hierarchies. Journal of Economic Theory, 90, 177-203.

Young, P. (1998) Social Structure and Individual Strategy. MIT Press. 